ROCKY MOUNTAIN

JOURNAL OF MATHEMATICS

Volume 33, Number 2, Summer 2003

\title{
ON THE TWO POINT PADÉ TABLE FOR A DISTRIBUTION
}

\author{
ELIANA X.L. DE ANDRADE AND JOHN H. MCCABE
}

\begin{abstract}
Some additional recurrence relations for the denominator polynomials of two point Padé approximants are derived. An example in which the coefficients of one of the two series, from which the Padé approximants are derived, are moments of a distribution is considered. For this example, properties of the denominator polynomials, and their zeros, are described.
\end{abstract}

1. Introduction. In this work we first look at some relations between the denominator polynomials of the Padé approximants and two point Padé approximants that are derived from the two series expansions

$$
\frac{\mu_{0}}{z}+\frac{\mu_{1}}{z^{2}}+\frac{\mu_{2}}{z^{3}}+\cdots+\frac{\mu_{k}}{z^{k+1}}+\cdots
$$

and

$$
-\mu_{-1}-\mu_{-2} z-\mu_{-3} z^{2}-\cdots-\mu_{-k} z^{k-1}-\cdots,
$$

where the $\mu_{k}, k=0, \pm 1, \pm 2, \ldots$, are real numbers. We then look at some properties of the polynomials when the coefficients of the series (1) are the moments of a particular distribution and those of series (2), though chosen in a natural way, are not moments of the distribution.

It is well known that if the coefficients $\mu_{k}, k=0, \pm 1, \pm 2, \ldots$, are such that the Hankel determinants of order $n$

$$
H_{n}^{(m)}=\left|\begin{array}{cccc}
\mu_{m} & \mu_{m+1} & \cdots & \mu_{m+n-1} \\
\mu_{m+1} & \mu_{m+2} & \cdots & \mu_{m+n} \\
\vdots & \vdots & \cdots & \vdots \\
\mu_{m+n-1} & \mu_{m+n} & \cdots & \mu_{m+2 n-2}
\end{array}\right|
$$

This research is supported by the Brazilian Science Foundation FAPESP under grant 00/07856-8.

Received by the editors on September 30, 2001, and in revised form on April 2, 2002 .

Copyright (C)2003 Rocky Mountain Mathematics Consortium 
are nonzero for $n=1,2, \ldots$ and $m=0, \pm 1, \pm 2, \ldots$, then there exist rational functions $A_{n}^{(r)}(z) / B_{n}^{(r)}(z)$ for $n=0,1, \ldots$ and $r=$ $0, \pm 1, \pm 2, \ldots$, such that

(4) $\frac{A_{n}^{(r)}(z)}{B_{n}^{(r)}(z)}=\left\{\begin{array}{r}\frac{\mu_{0}}{z}+\frac{\mu_{1}}{z^{2}}+\cdots+\frac{\mu_{n+r-1}}{z^{n+r}}+\text { lower order terms } \\ -\mu_{-1}-\mu_{-2} z-\cdots-\mu_{-n-r} z^{n-r-1} \\ + \text { higher order terms }\end{array}\right.$

when expanded accordingly.

These rational functions are conveniently displayed as the two point Padé table

(5)

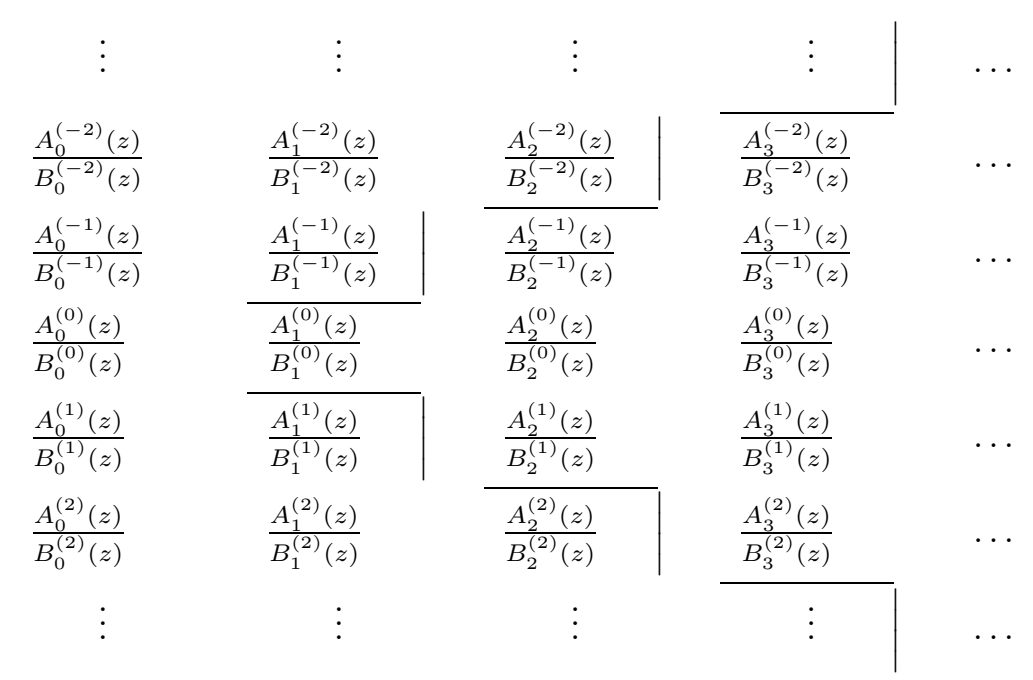

The elements between the staircase lines are strictly two point Padé approximants. That is, the entry

$$
\frac{A_{n}^{(r)}(z)}{B_{n}^{(r)}(z)}, \quad n=0,1,2, \ldots, \quad r=0, \pm 1, \pm 2, \ldots, \quad|r|<n,
$$

is a ratio of polynomials of degree $n-1$ and $n$ respectively and "fits" $n+r$ terms of the series (2) and $n-r$ terms of the series (2) when expanded accordingly. 
Those above this region, i.e., the elements

$$
\frac{A_{n}^{(r)}(z)}{B_{n}^{(r)}(z)}, \quad n=0,1,2, \ldots,-r, \quad r=-1,-2,-3, \ldots,
$$

are straightforward Padé approximants for the series (2). Thus, for $n=0,1, \cdots, r$ and $r=1,2, \cdots$

$$
\frac{A_{n}^{(r)}(z)}{B_{n}^{(r)}(z)}=P_{n,-r-1}(z)=\frac{\sum_{j=0}^{-r-1} a_{j} z^{j}}{\sum_{j=0}^{n} b_{j} z^{j}} .
$$

Those below the lower staircase line are the elements of Wynn's E array for the series (1). Thus,

$$
\frac{A_{n}^{(r)}(z)}{B_{n}^{(r)}(z)}=E_{n, r-n}(z)=z^{n-r} \frac{\sum_{j=0}^{r-1} c_{j} z^{j}}{\sum_{j=0}^{n} d_{j} z^{j}},
$$

for $n=0,1, \cdots, r$ and $r=1,2, \cdots$. The rational functions in any ordered sequence, horizontal, diagonal or staircase, for instance, are the convergents of continued fractions associated with the two series. For example, those in the center row $\frac{A_{n}^{(0)}(z)}{B_{n}^{(0)}(z)}, n=1,2, \ldots$, are the convergents of a continued fraction of the form

$$
\frac{\mu_{0}}{z-\beta_{1}^{(0)}}-\frac{\alpha_{2}^{(0)} z}{z-\beta_{2}^{(0)}}-\frac{\alpha_{3}^{(0)} z}{z-\beta_{3}^{(0)}}-\frac{\alpha_{4}^{(0)} z}{z-\beta_{4}^{(0)}}-\ldots,
$$

The coefficients of this and other continued fractions can be generated by the $q-d$ algorithm as follows.

Algorithm 1. For $r=\ldots,-2,-1,0,1,2, \ldots$, set

$$
\alpha_{1}^{(r)}=0, \quad \beta_{1}^{(r)}=\frac{\mu_{r}}{\mu_{r-1}}
$$

Then, for $n=2,3, \ldots$, and for $r=\ldots,-2,-1,0,1,2, \ldots$, set

$$
\begin{aligned}
& \alpha_{n}^{(r)}=\beta_{n-1}^{(r+1)}+\alpha_{n-1}^{(r+1)}-\beta_{n-1}^{(r)} \\
& \beta_{n}^{(r)}=\frac{\alpha_{n}^{(r)} \beta_{n-1}^{(r-1)}}{\alpha_{n}^{(r-1)}}
\end{aligned}
$$


The coefficients are conveniently displayed in the $\alpha-\beta$ array

$\begin{array}{ccccccc}\vdots & \vdots & \vdots & \vdots & \vdots & \vdots & \ldots \\ \beta_{1}^{(-2)} & \alpha_{2}^{(-2)} & \beta_{2}^{(-2)} & \alpha_{3}^{(-2)} & \beta_{3}^{(-2)} & \alpha_{4}^{(-2)} & \ldots \\ \beta_{1}^{(-1)} & \alpha_{2}^{(-1)} & \beta_{2}^{(-1)} & \alpha_{3}^{(-1)} & \beta_{3}^{(-1)} & \alpha_{4}^{(-1)} & \ldots \\ \beta_{1}^{(0)} & \alpha_{2}^{(0)} & \beta_{2}^{(0)} & \alpha_{3}^{(0)} & \beta_{3}^{(0)} & \alpha_{4}^{(0)} & \ldots \\ \beta_{1}^{(1)} & \alpha_{2}^{(1)} & \beta_{2}^{(1)} & \alpha_{3}^{(1)} & \beta_{3}^{(1)} & \alpha_{4}^{(1)} & \ldots \\ \beta_{1}^{(2)} & \alpha_{2}^{(2)} & \beta_{2}^{(2)} & \alpha_{3}^{(2)} & \beta_{3}^{(2)} & \alpha_{4}^{(2)} & \ldots \\ \vdots & \vdots & \vdots & \vdots & \vdots & \vdots & \ldots\end{array}$

and those in any row are the coefficients of a continued fraction, of the same form as (6) but with a partial sum of one of the series added at the beginning. The convergents form the corresponding row of the two point Padé table (5).

For any given value of $r$ the polynomials $B_{n}^{(r)}(z), n=0,1,2, \ldots$, are the denominators of the convergents of the continued fraction

$$
\frac{\mu_{r}}{z-\beta_{1}^{(r)}}-\frac{\alpha_{2}^{(r)} z}{z-\beta_{2}^{(r)}}-\frac{\alpha_{3}^{(r)} z}{z-\beta_{3}^{(r)}}-\frac{\alpha_{4}^{(r)} z}{z-\beta_{4}^{(r)}}-
$$

and they are conveniently displayed in the following table

$$
\begin{array}{lllll}
B_{0}^{(-2)}(z) & B_{1}^{(-2)}(z) & B_{2}^{(-2)}(z) & B_{3}^{(-2)}(z) & \ldots \\
B_{0}^{(-1)}(z) & B_{1}^{(-1)}(z) & B_{2}^{(-1)}(z) & B_{3}^{(-1)}(z) & \ldots \\
B_{0}^{(0)}(z) & B_{1}^{(0)}(z) & B_{2}^{(0)}(z) & B_{3}^{(0)}(z) & \ldots \\
B_{0}^{(1)}(z) & B_{1}^{(1)}(z) & B_{2}^{(1)}(z) & B_{3}^{(1)}(z) & \ldots \\
B_{0}^{(2)}(z) & B_{1}^{(2)}(z) & B_{2}^{(2)}(z) & B_{3}^{(2)}(z) & \cdots
\end{array}
$$

The denominator polynomials and the numerator polynomials of the rational functions in the two point Padé table satisfy various three 
term recurrence relations involving any three neighboring polynomials. Such recurrence relations appear throughout the literature and many are given in [7] where they are developed from an alternative form of the usual quotient difference algorithm for transforming a single power series into its Padé table. However, corresponding results hold in the two point Padé table and, of course, the whole of one point Padé for the series (2) or the E array for the series (1) can be derived from the above algorithm by setting values for particular $\alpha$ 's or $\beta$ 's instead of calculating them from the algorithm. Some useful recurrence relations for trios of polynomials selected from the five rational functions that are linked by Wynn's identity, namely those in the positions shown diagrammatically by

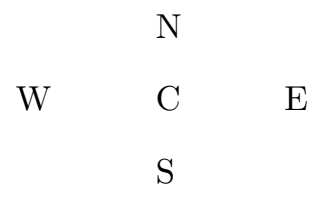

are given below in terms of the denominators $B_{n}^{(r)}(z)$. Thus, for the five polynomials

$$
\begin{aligned}
& B_{n}^{(r-1)}(z) \\
& B_{n-1}^{(r)}(z) \quad B_{n}^{(r)}(z) \quad B_{n+1}^{(r)}(z) \\
& B_{n}^{(r+1)}(z)
\end{aligned}
$$

we have

$$
\begin{array}{ll}
B_{n+1}^{(r)}(z)=\left(z-\beta_{n+1}^{(r)}\right) B_{n}^{(r)}(z)-\alpha_{n+1}^{(r)} z B_{n-1}^{(r)}(z) & \bullet \bullet \bullet \\
B_{n}^{(r+1)}(z)=\frac{1}{z}\left(B_{n+1}^{(r)}(z)+\beta_{n+1}^{(r)} B_{n}^{(r)}(z)\right) & \bullet \bullet \\
B_{n}^{(r+1)}(z)=B_{n}^{(r)}(z)-\alpha_{n+1}^{(r)} B_{n-1}^{(r)}(z) & \bullet
\end{array}
$$




$$
\begin{aligned}
& B_{n}^{(r-1)}(z)=\frac{\left(z+\beta_{n+1}^{(r)}\right) B_{n}^{(r)}(z)-z B_{n}^{(r+1)}(z)}{\alpha_{n+1}^{(r)}+\beta_{n+1}^{(r)}} \\
& B_{n}^{(r-1)}(z)=\frac{z B_{n}^{(r)}(z)-B_{n+1}^{(r)}(z)}{\alpha_{n+1}^{(r)}+\beta_{n+1}^{(r)}} \\
& B_{n}^{(r-1)}(z)=\frac{\beta_{n+1}^{(r)} B_{n}^{(r)}(z)+\alpha_{n+1}^{(r)} z B_{n-1}^{(r)}(z)}{\alpha_{n+1}^{(r)}+\beta_{n+1}^{(r)}}
\end{aligned}
$$

2. Triangular sequences. The denominators linked by (12) form a triangle in which each polynomial can be expressed as a linear combination of the other two. We now extend this to the polynomials that form the triangles constructed by the polynomials $B_{n}^{(r)}(z), B_{n}^{(r+s)}(z)$ and $B_{n-s}^{(r)}(z)$. This triangle is shown below.

$$
\begin{array}{ccccc}
B_{n-s}^{(r)}(z) & B_{n-s+1}^{(r)}(z) & \cdots & \cdots & B_{n}^{(r)}(z) \\
& B_{n-s+1}^{(r+1)}(z) & \cdots & \cdots & B_{n}^{(r+1)}(z) \\
& & \ddots & & \vdots \\
& & & \ddots & \vdots \\
& & & & B_{n}^{(r+s)}(z)
\end{array}
$$

Then, the polynomials of each vertex of the triangle can be written as a linear combination of the polynomials on the opposite side. Specifically, we have the following results.

Theorem 1. (i) The polynomial $B_{n}^{(r)}(z)$ can be written as

$$
B_{n}^{(r)}(z)=\sum_{i=0}^{s} \lambda_{n, i}^{(r+s, s)} B_{n-i}^{(r+s-i)}(z)
$$


where

$$
\begin{aligned}
& \lambda_{n, i}^{(r+s, s)}=\lambda_{n, i}^{(r+s, s-1)}+\alpha_{n+1}^{(r)} \lambda_{n-1, i-1}^{(r+s-1, s-1)}, \quad i=0,1, \ldots, s, \\
& \lambda_{n, 0}^{(k, 0)}=1 \text { and } \lambda_{n,-1}^{(k, m)}=\lambda_{n, s}^{(k, m-1)}=0 . \\
& \text { Alternatively, for } i=0,1, \cdots, s
\end{aligned}
$$$$
\lambda_{n, i}^{(r, s)}=\sum_{j_{1}=i}^{s} \sum_{j_{2}=i}^{j_{1}} \cdots \sum_{j_{i}=i}^{j_{i-1}} \prod_{k=1}^{i} \alpha_{n-(k-2)}^{\left(r+s-j_{k}\right)} .
$$

(ii) The polynomial $B_{n}^{(r+s)}(z)$ satisfy

$$
B_{n}^{(r+s)}(z)=\sum_{i=0}^{s} \delta_{n, i}^{(r, s)} B_{n-i}^{(r)}(z)
$$

where

$$
\delta_{n, i}^{(r, s)}=\delta_{n, i}^{(r, s-1)}-\alpha_{n+1}^{(r+s-1)} \delta_{n-1, i-1}^{(r, s-1)}, \quad i=0,1, \ldots, s
$$

$\delta_{n, 0}^{(r, 0)}=1$ and $\delta_{n,-1}^{(r, s)}=\delta_{n, s}^{(r, s-1)}=0$.

Alternatively, for $i=0,1, \cdots, s$

$$
\delta_{n, i}^{(r, s)}=(-1)^{i} \sum_{j_{1}=i}^{s} \sum_{j_{2}=i-1}^{j_{1}-1} \sum_{j_{3}=i-2}^{j_{2}-1} \ldots \sum_{j_{i}=1}^{j_{i-1}-1} \prod_{k=1}^{i} \alpha_{n-(k-2)}^{\left(r+j_{k}-1\right)}
$$

(iii) Finally, for the polynomial $B_{n-s}^{(r)}(z)$ we have

$$
B_{n-s}^{(r)}(z)=\sum_{i=0}^{s} \gamma_{n, i}^{(r, s)} B_{n}^{(r+i)}(z)
$$

where

$$
\gamma_{n, i}^{(r, s)}=\frac{\gamma_{n, i}^{(r, s-1)}-\gamma_{n, i-1}^{(r+1, s-1)}}{\alpha_{n+2-s}^{(r)}}, \quad i=0,1, \ldots, s,
$$

with $\gamma_{n, 0}^{(r, 0)}=\gamma_{n, 0}^{(r+1,0)}=1$ and $\gamma_{n,-1}^{(r, s-1)}=\gamma_{n, s}^{(r, s-1)}=0$. 
Alternatively, for $i=0,1, \cdots, s$

$$
\gamma_{n, i}^{(r, s)}=\frac{(-1)^{i}}{\alpha_{n-s+2}^{(r)}} \sum_{\substack{j_{\mu}=j_{\mu-1}-1 \\ \mu=2, \ldots, s-i}}^{j_{\mu-1}} \sum_{\substack{j_{\mu}=j_{\mu-1}-1 \\ \mu=s-i+1, \ldots, s-1}}^{s-\mu} \prod_{k=1}^{s-1} \frac{1}{\alpha_{n-k+2}^{\left(r+j_{k}\right)}},
$$

where $j_{1}=i$ and $j_{1} \geq j_{2} \geq \cdots \geq j_{s-1} \geq 0$.

Proof. Consider first the results in (i).

Observe that for $s=1$ the equations in (i) are given by

$$
B_{n}^{(r)}(z)=\lambda_{n, 0}^{(r, 1)} B_{n}^{(r+1)}(z)+\lambda_{n, 1}^{(r, 1)} B_{n-1}^{(r)}(z)
$$

where $\lambda_{n, 0}^{(r, 1)}=1$ and $\lambda_{n, 1}^{(r, 1)}=\alpha_{n+1}^{(r)}$.

Then,

$$
B_{n}^{(r+1)}(z)=B_{n}^{(r)}(z)-\alpha_{n+1}^{(r)} B_{n-1}^{(r)}(z),
$$

the same result given by equation (12).

From equation (12) we obtain

$$
B_{n}^{(r-1)}(z)=B_{n}^{(r)}(z)+\alpha_{n+1}^{(r-1)} B_{n-1}^{(r-1)}(z)=\hat{\lambda}_{n, 0}^{(r, 1)} B_{n}^{(r)}(z)+\hat{\lambda}_{n, 1}^{(r, 1)} B_{n-1}^{(r-1)}(z) .
$$

So, $\hat{\lambda}_{n, 0}^{(r, 1)}=1$ and $\hat{\lambda}_{n, 1}^{(r, 1)}=\alpha_{n+1}^{(r-1)}$.

Suppose now $s \leq n$ and

$$
B_{n}^{(r-m)}(z)=\sum_{i=0}^{m} \hat{\lambda}_{n, i}^{(r, m)} B_{n-i}^{(r-i)}(z), \quad m=1, \ldots, s-1,
$$

where for $i=0,1, \ldots, m$,

$$
\begin{aligned}
\hat{\lambda}_{n, i}^{(r, m)} & =\hat{\lambda}_{n, i}^{(r, m-1)}+\alpha_{n+1}^{(r-m)} \hat{\lambda}_{n-1, i-1}^{(r-1, m-1)} \\
& =\sum_{j_{1}=i}^{m} \sum_{j_{2}=i}^{j_{1}} \cdots \sum_{j_{i}=i}^{j_{i-1}} \prod_{k=1}^{i} \alpha_{n-(k-2)}^{\left(r-j_{k}\right)} .
\end{aligned}
$$


So, from (12) and (17),

$$
\begin{aligned}
B_{n}^{(r-m-1)}(z)= & B_{n}^{(r-m)}(z)+\alpha_{n+1}^{(r-m-1)} B_{n-1}^{(r-m-1)}(z) \\
= & \sum_{i=0}^{m} \hat{\lambda}_{n, i}^{(r, m)} B_{n-i}^{(r-i)}(z)+\alpha_{n+1}^{(r-m-1)} \sum_{i=0}^{m} \hat{\lambda}_{n-1, i}^{(r-1, m)} B_{n-1-i}^{(r-1-i)}(z) \\
= & \hat{\lambda}_{n, 0}^{(r, m)} B_{n}^{(r)}(z)+\sum_{i=1}^{m}\left(\hat{\lambda}_{n, i}^{(r, m)}+\alpha_{n+1}^{(r-m-1)} \hat{\lambda}_{n-1, i-1}^{(r-1, m)}\right) \\
& \cdot B_{n-i}^{(r-i)}(z)+\alpha_{n+1}^{(r-m-1)} \hat{\lambda}_{n-1, m}^{(r-1, m)} B_{n-m-1}^{(r-m-1)}(z) \\
= & \sum_{i=0}^{m+1} \hat{\lambda}_{n, i}^{(r, m+1)} B_{n-i}^{(r-i)}(z),
\end{aligned}
$$

where

$$
\begin{aligned}
& \hat{\lambda}_{n, 0}^{(r, m+1)}=\hat{\lambda}_{n, 0}^{(r, m)} \\
& \hat{\lambda}_{n, i}^{(r, m+1)}=\hat{\lambda}_{n, i}^{(r, m)}+\alpha_{n+1}^{(r-m-1)} \hat{\lambda}_{n-1, i-1}^{(r-1, m)}, \quad i=1, \ldots, m \\
& \hat{\lambda}_{n, m+1}^{(r, m+1)}=\alpha_{n+1}^{(r-m-1)} \hat{\lambda}_{n-1, m}^{(r-1, m)} .
\end{aligned}
$$

Thus, we can write

$$
\hat{\lambda}_{n, i}^{(r, m+1)}=\hat{\lambda}_{n, i}^{(r, m)}+\alpha_{n+1}^{(r-m-1)} \hat{\lambda}_{n-1, i-1}^{(r-1, m)}, \quad i=0, \ldots, m+1,
$$

with $\hat{\lambda}_{n, 0}^{(r, 0)}=1$ and $\hat{\lambda}_{n,-1}^{(r, m)}=\hat{\lambda}_{n, m+1}^{(r, m)}=0$.

Now, from (18) and (19) we have

$$
\begin{aligned}
\hat{\lambda}_{n, i}^{(r, m+1)}= & \sum_{j_{1}=i}^{m} \sum_{j_{2}=i}^{j_{1}} \ldots \sum_{j_{i}=i}^{j_{i-1}} \prod_{k=1}^{i} \alpha_{n-(k-2)}^{\left(r-j_{k}\right)} \\
& +\alpha_{n+1}^{(r-m-1)} \sum_{j_{1}=i-1}^{m} \sum_{j_{2}=i-1}^{j_{1}} \ldots \sum_{j_{i-1}=i-1}^{j_{i-2}} \prod_{k=1}^{i-1} \alpha_{n-1-(k-2)}^{\left(r-1-j_{k}\right)} \\
= & \sum_{j_{1}=i}^{m} \sum_{j_{2}=i}^{j_{1}} \ldots \sum_{j_{i}=i}^{j_{i-1}} \prod_{k=1}^{i} \alpha_{n-(k-2)}^{\left(r-j_{k}\right)} \\
& +\sum_{j_{0}=m}^{m} \sum_{j_{1}=i-1}^{m} \sum_{j_{2}=i-1}^{j_{1}} \ldots \sum_{j_{i-1}=i-1}^{j_{i-2}} \prod_{k=0}^{i-1} \alpha_{n-1-(k-2)}^{\left(r-1-j_{k}\right)}
\end{aligned}
$$




$$
\begin{aligned}
& =\sum_{j_{1}=i}^{m} \sum_{j_{2}=i}^{j_{1}} \cdots \sum_{j_{i}=i}^{j_{i-1}} \prod_{k=1}^{i} \alpha_{n-(k-2)}^{\left(r-j_{k}\right)}+\sum_{j_{1}=m+1}^{m+1} \sum_{j_{2}=i}^{j_{1}} \cdots \sum_{j_{i}=i}^{j_{i-1}} \prod_{k=1}^{i} \alpha_{n-(k-2)}^{\left(r-j_{k}\right)} \\
& =\sum_{j_{1}=i}^{m+1} \sum_{j_{2}=i}^{j_{1}} \cdots \sum_{j_{i}=i}^{j_{i-1}} \prod_{k=1}^{i} \alpha_{n-(k-2)}^{\left(r-j_{k}\right)}, \quad i=0, \ldots, m+1
\end{aligned}
$$

So, by mathematical induction, it follows that

$$
B_{n}^{(r-s)}(z)=\sum_{i=0}^{s} \hat{\lambda}_{n, i}^{(r, s)} B_{n-i}^{(r-i)}(z), \quad s=1, \ldots, n
$$

where

$$
\hat{\lambda}_{n, i}^{(r, s)}=\hat{\lambda}_{n, i}^{(r, s-1)}+\alpha_{n+1}^{(r-s)} \hat{\lambda}_{n-1, i-1}^{(r-1, s-1)}, \quad i=0,1, \ldots, s,
$$

with $\hat{\lambda}_{n, 0}^{(r, 0)}=1$ and $\hat{\lambda}_{n,-1}^{(r, s)}=\hat{\lambda}_{n, s}^{(r, s-1)}=0$.

Also,

$$
\hat{\lambda}_{n, i}^{(r, s)}=\sum_{j_{1}=i}^{s} \sum_{j_{2}=i}^{j_{1}} \cdots \sum_{j_{i}=i}^{j_{i-1}} \prod_{k=1}^{i} \alpha_{n-(k-2)}^{\left(r-j_{k}\right)}, \quad i=0,1, \ldots, s .
$$

Setting $r-s=r$ in the equations (20), (21) and (22) we find the results in (i).

Using again the equation (12), we have

$$
B_{n-1}^{(r)}(z)=\frac{1}{\alpha_{n+1}^{(r)}}\left(B_{n}^{(r)}(z)-B_{n}^{(r+1)}(z)\right) .
$$

Thus, from (12) and the above equation, the results in (ii) and (iii) follow by mathematical induction.

3. Extension of a distribution. In the particular case where the coefficients of the series (1) and (2) are the moments of a strong distribution $d \psi(t)$, i.e.,

$$
\mu_{n}=\int_{a}^{b} t^{n} d \psi(t), \quad n=0, \pm 1, \pm, 2, \ldots,
$$


for $(a, b) \subset \Re$, the results in (i) allow us to express the denominator polynomials $B_{n}^{(r)}(z), r=0,1,2, \ldots,(n-1)$, in terms of the classical orthogonal polynomials associated with the distribution. Thus, for example, the denominators on the central row of the table (9) can be written as

$$
B_{n}^{(0)}(z)=\sum_{i=0}^{n} \lambda_{n, i}^{(0, n)} B_{n-i}^{(n-i)}(z) .
$$

See, for example, $[\mathbf{1}],[\mathbf{2}],[\mathbf{8}]$ and $[\mathbf{9}]$ for some details of such results.

The classical orthogonal polynomials are themselves the denominators of the successive convergents of a continued fraction which can be written in terms of the coefficients in the table (8). Specifically, the sequence $B_{1}^{(1)}(z), B_{2}^{(2)}(z), B_{3}^{(3)}(z), B_{4}^{(4)}(z), \ldots$ are the denominators of the continued fraction

$$
\frac{\alpha_{0}}{z-\beta_{1}^{(1)}}-\frac{\left(\alpha_{2}^{(1)}+\beta_{2}^{(1)}\right) \alpha_{2}^{(0)}}{z-\left(\alpha_{2}^{(1)}+\beta_{2}^{(1)}+\alpha_{3}^{(1)}\right)}-\frac{\left(\alpha_{3}^{(2)}+\beta_{3}^{(2)}\right) \alpha_{3}^{(1)}}{z-\left(\alpha_{3}^{(2)}+\beta_{3}^{(2)}+\alpha_{4}^{(2)}\right)}-\ldots
$$

It follows that the table of coefficients provides the standard three term recurrence relation for the orthogonal polynomials, namely,

$$
\begin{aligned}
B_{n+1}^{(n+1)}(z)= & {\left[z-\left(\alpha_{n+1}^{(n)}+\beta_{n+1}^{(n)}+\alpha_{n+2}^{(n)}\right)\right] B_{n}^{(n)}(z) } \\
& -\left(\alpha_{n+1}^{(n)}+\beta_{n+1}^{(n)}\right) \alpha_{n+1}^{(n-1)} B_{n-1}^{(n-1)}(z) .
\end{aligned}
$$

These results are obtained by using (14) and (12) alternately to provide the continued fraction whose denominators are $B_{1}^{(0)}(z), B_{1}^{(1)}(z)$, $B_{2}^{(1)}(z), B_{2}^{(2)}(z), B_{3}^{(2)}(z), B_{3}^{(3)}(z), B_{4}^{(3)}(z), \ldots$ and then taking the even contraction to give (23).

In a similar way we can derive the recurrence relation for the denominators forming any diagonal in the table, namely $B_{n}^{(n+s)}(z)$, $s=0, \pm 1, \pm 2, \pm 3, \ldots$. We find

$$
\begin{aligned}
B_{n+1}^{(n+1+s)}(z)= & {\left[z-\left(\alpha_{n+1}^{(n+s)}+\beta_{n+1}^{(n+s)}+\alpha_{n+2}^{(n+s)}\right)\right] B_{n}^{(n+s)}(z) } \\
& -\left(\alpha_{n+1}^{(n+s)}+\beta_{n+1}^{(n+s)}\right) \alpha_{n+1}^{(n-1+s)} B_{n-1}^{(n-1+s)}(z) .
\end{aligned}
$$

Many of the details of these results can be found in $[\mathbf{1}]$. 
It does not have to be the case that the coefficients of (1) and (2) are the moments of a strong distribution. The tables of coefficients and denominators will exist provided that the conditions (3) hold, but of course there will be no orthogonality. An interesting half way situation is when the coefficients of the series (1) are moments of a distribution while those in (2) are chosen in some other way. That is, the above definition of $\mu_{n}$ holds only for non-negative values of $n$. The following is an example of such a case.

Let us consider the distribution

$$
d \psi(t)=\frac{1}{\sqrt{2 \pi}} t^{-1 / 2} e^{-t / 2} d t \quad \text { on }[0, \infty),
$$

and the moments defined by

$$
\mu_{n}=\frac{1}{\sqrt{2 \pi}} \int_{0}^{\infty} t^{n-1 / 2} e^{-t / 2} d t, \quad n=0,1, \ldots
$$

Using the change of variable $s=t / 2$ in the above integral we obtain

$$
\mu_{n}=\frac{2^{n}}{\sqrt{\pi}} \int_{0}^{\infty} s^{n-1 / 2} e^{-s} d s=\frac{2^{n}}{\sqrt{\pi}} \Gamma\left(n+\frac{1}{2}\right), \quad n=0,1, \ldots,
$$

where $\Gamma(z)$ is the Gamma function.

Using well-known properties of the Gamma function,

$$
\Gamma(z+1)=z \Gamma(z) \quad \text { and } \quad \Gamma(1 / 2)=\sqrt{\pi},
$$

we obtain $\mu_{0}=1$ and for $n=1,2, \ldots$,

$$
\Gamma\left(n+\frac{1}{2}\right)=(2 n-1)(2 n-3) \cdots(1) \frac{\sqrt{\pi}}{2^{n}} .
$$

Hence, we have proved the following result

Theorem 2. For $n=0,1,2, \ldots$, the moments are given by

$$
\mu_{n}=\frac{1}{\sqrt{2 \pi}} \int_{0}^{\infty} t^{n-1 / 2} e^{-t / 2} d t=(2 n-1) ! !
$$


where $(2 n-1) ! !:=(2 n-1)(2 n-3) \cdots(3)(1), n=1,2,3, \ldots$, and $(-1) ! !:=1$.

Another property of the Gamma function is

$$
\Gamma\left(z+\frac{1}{2}\right) \Gamma\left(-z+\frac{1}{2}\right)=\frac{\pi}{\cos (\pi z)} .
$$

Then, we obtain

$$
\Gamma\left(n+\frac{1}{2}\right) \Gamma\left(-n+\frac{1}{2}\right)=\frac{\pi}{\cos (\pi n)}=\frac{\pi}{(-1)^{n}}, \quad n=1,2, \ldots
$$

So we can define, in a natural way,

$$
\mu_{-n}=\frac{(-1)^{n}}{(2 n-1) ! !}=:(-(2 n+1)) ! !, \quad n=1,2, \ldots
$$

By mathematical induction and using the equations in Algorithm 1 we can prove the following result.

Theorem 3. Let $\mu_{n}, n=0, \pm 1, \pm 2, \ldots$, be defined by (26) and (27). Then the coefficients of the table (8) are given by

$$
\beta_{n+1}^{(r)}=2 r-2 n-1 \quad \text { and } \quad \alpha_{n+1}^{(r)}=2 n, \quad n \geq 0 .
$$

Hence, the $\alpha-\beta$ array is

\begin{tabular}{r|ccccccccc}
$r$ & $\beta_{1}^{(r)}$ & $\alpha_{2}^{(r)}$ & $\beta_{2}^{(r)}$ & $\alpha_{3}^{(r)}$ & $\beta_{3}^{(r)}$ & $\alpha_{4}^{(r)}$ & $\beta_{4}^{(r)}$ & $\alpha_{5}^{(r)}$ & $\ldots$ \\
\hline$\vdots$ & $\vdots$ & $\vdots$ & $\vdots$ & $\vdots$ & $\vdots$ & $\vdots$ & $\vdots$ & $\vdots$ & $\ldots$ \\
-2 & -5 & 2 & -7 & 4 & -9 & 6 & -11 & 8 & $\ldots$ \\
-1 & -3 & 2 & -5 & 4 & -7 & 6 & -9 & 8 & $\ldots$ \\
0 & -1 & 2 & -3 & 4 & -5 & 6 & -7 & 8 & $\ldots$ \\
1 & 1 & 2 & -1 & 4 & -3 & 6 & -5 & 8 & $\ldots$ \\
2 & 3 & 2 & 1 & 4 & -1 & 6 & -3 & 8 & $\ldots$ \\
$\vdots$ & $\vdots$ & $\vdots$ & $\vdots$ & $\vdots$ & $\vdots$ & $\vdots$ & $\vdots$ & $\vdots$ & $\ldots$
\end{tabular}


From (26) and (27) we have the two series

$$
\frac{1}{z}+\frac{1}{z^{2}}+\frac{3}{z^{3}}+\frac{15}{z^{4}}+\frac{105}{z^{5}}+\cdots
$$

and

$$
1-\frac{1}{3} z+\frac{1}{15} z^{2}-\frac{1}{105} z^{3}+\cdots .
$$

These series were studied by McCabe in [4] and [5]. They are related, respectively, to the MacLauren series and an asymptotic expansion for Dawson's integral

$$
e^{-z^{2}} \int_{0}^{z} e^{t^{2}} d t
$$

3.1 The denominator polynomials. The recurrence relation (24) and the definitions (28) hold for this example. Hence,

$\beta_{n+1}^{(n+s)}+\alpha_{n+1}^{(n+s)}+\alpha_{n+2}^{(n+s)}=2(n+s)-2 n-1+2 n+2(n+1)=4 n+2 s+1$

and

$\left(\alpha_{n+1}^{(n+s)}+\beta_{n+1}^{(n+s)}\right) \alpha_{n+1}^{(n-1+s)}=[2 n+2(n+s)-2 n-1] 2 n=2 n(2 n+2 s-1)$.

We have the following result.

Theorem 4. The polynomials $B_{n}^{(n+s)}(z), s=0, \pm 1, \pm 2, \ldots$, satisfy the recurrence relation

$$
\begin{aligned}
B_{n+1}^{(n+1+s)}(z)= & {[z-(4 n+2 s+1)] B_{n}^{(n+s)}(z) } \\
& -2 n(2 n+2 s-1) B_{n-1}^{(n-1+s)}(z), \quad n \geq 1
\end{aligned}
$$

with $B_{0}^{(s)}(z)=1$ and $B_{1}^{(1+s)}(z)=z-(2 s+1)$.

It is well known that the Laguerre polynomials, $L_{n}^{(\alpha)}(x), n \geq 0$, are orthogonal with respect to the inner product

$$
\langle f, g\rangle=\int_{0}^{\infty} x^{\alpha} e^{-x} f(x) g(x) d x, \quad \alpha>-1 .
$$


We refer to Chihara [3] and Szegö $[\mathbf{1 0}]$ for more about these polynomials.

In the monic form these polynomials are defined by the Rodrigues Formula

$$
\hat{L}_{n}^{(\alpha)}(x)=(-1)^{n} x^{-\alpha} e^{x} \frac{d^{n}}{d x^{n}}\left[x^{\alpha+n} e^{-x}\right]
$$

and they satisfy the recurrence relation

$$
\hat{L}_{n+1}^{(\alpha)}(x)=[x-(2 n+\alpha+1)] \hat{L}_{n}^{(\alpha)}(x)-n(n+\alpha) \hat{L}_{n-1}^{(\alpha)}(x), \quad n \geq 1,
$$

with $\hat{L}_{0}^{(\alpha)}(x)=1$ and $\hat{L}_{1}^{(\alpha)}(x)=x-(\alpha+1)$.

Setting $x=z / 2$ and $\alpha=s-1 / 2$ in the equation (31) we obtain for $n \geq 1$

$$
\begin{aligned}
2^{n+1} \hat{L}_{n+1}^{(s-1 / 2)}(z / 2)= & {[z-(4 n+2 s+1)] 2^{n} \hat{L}_{n}^{(s-1 / 2)}(z / 2) } \\
& -2 n(2 n+2 s-1) 2^{n-1} \hat{L}_{n-1}^{(s-1 / 2)}(z / 2) .
\end{aligned}
$$

Observe that $\hat{L}_{0}^{(s-1 / 2)}(z / 2)=1,2 \hat{L}_{1}^{(s-1 / 2)}(z / 2)=z-(2 s+1)$ and, for $s=0,1,2, \ldots$, we have $\alpha>-1$.

Hence, from (29) and (32),

$$
B_{n}^{(n+s)}(z)=2^{n} \hat{L}_{n}^{(s-1 / 2)}(z / 2), \quad s=0,1,2, \ldots .
$$

Using Leibnitz's Rule in the equation (30) and setting $x=z / 2$ and $\alpha=s-1 / 2$ for $s=0,1,2, \ldots$, we obtain

$$
\begin{aligned}
B_{n}^{(n+s)}(z)= & \sum_{k=0}^{n}(-1)^{k} 2^{k}\left(\begin{array}{l}
n \\
k
\end{array}\right)(n+s-1 / 2)(n+s-3 / 2) \cdots \\
& \cdot(n+s-k+1 / 2) z^{n-k} \\
= & \sum_{i=0}^{n}(-1)^{n-i}\left(\begin{array}{c}
n \\
i
\end{array}\right) \frac{(2(n+s)-1) ! !}{(2(s+i)-1) ! !} z^{i} .
\end{aligned}
$$

Thus, for $r=n, n+1, \ldots$, the polynomials $B_{n}^{(r)}(z)$ can be written as

$$
B_{n}^{(r)}(z)=\sum_{i=0}^{n}(-1)^{n-i}\left(\begin{array}{c}
n \\
i
\end{array}\right) \frac{(2 r-1) ! !}{(2(r-n+i)-1) ! !} z^{i} .
$$


From equations $(12),(28)$ and $(33)$, and noting that $(-(2 n+1)) ! !=$ $(-1)^{n} /(2 n-1) ! !$, we obtain

$$
\begin{aligned}
B_{n}^{(n-1)}(z) & =B_{n}^{(n)}(z)+2 n B_{n-1}^{(n-1)}(z) \\
& =z^{n}+\sum_{i=0}^{n-1}(-1)^{n-i}\left(\begin{array}{c}
n \\
i
\end{array}\right) \frac{(2 n-3) ! !}{(2 i-3) ! !} z^{i} .
\end{aligned}
$$

Observe that this is exactly the equation (33) for $r=n-1$. Now, given that

$$
B_{n}^{(n-s)}(z)=B_{n}^{(n+1-s)}(z)+2 n B_{n-1}^{(n-s)}(z),
$$

then by mathematical induction we can prove that the equation (33) also holds for $r=n-1, n-2, \ldots$.

For the distribution $d \psi(t)$ given by $(25)$ the coefficients of the recurrence relation are given by equations in (28). Thus,

$$
\alpha_{n-(k-2)}^{\left(n-j_{k}\right)}=2(n-k+1) .
$$

Then, setting $r=n-s$ in (16) we can write the polynomials $B_{n}^{(n-s)}(z)$, $s=0,1, \ldots, n$, as the following linear combination of the classical orthogonal polynomials $B_{n-s}^{(n-s)}(z), \ldots, B_{n-1}^{(n-1)}(z), B_{n}^{(n)}(z)$,

$$
B_{n}^{(n-s)}(z)=\sum_{i=0}^{s} 2^{i}\left(\begin{array}{c}
s \\
i
\end{array}\right) \frac{n !}{(n-i) !} B_{n-i}^{(n-i)}(z), \quad s=0,1, \ldots, n .
$$

Theorem 5. The polynomials $B_{n}^{(r)}(z), n \geq 1, r=0 \pm 1, \pm 2, \ldots$, associated to the distribution $d \psi(t)$ given by (25), satisfy

$$
\frac{d}{d z}\left(B_{n}^{(r)}(z)\right)=n B_{n-1}^{(r)}(z) .
$$

This theorem is easily proved by differentiating the equation (33).

From (35) we further obtain

$$
\frac{d^{2} B_{n}^{(r)}(z)}{d z^{2}}=n \frac{d B_{n-1}^{(r)}(z)}{d z}=n(n-1) B_{n-2}^{(r)}(z), \quad n \geq 2 .
$$


Using the equations in (28) and the above equality in the recurrence relation $(10)$, we see that

$$
B_{n}^{(r)}(z)=(z-2 r+2 n-1) \frac{1}{n}\left(\frac{d B_{n}^{(r)}(z)}{d z}\right)-\frac{2 z}{n}\left(\frac{d^{2} B_{n}^{(r)}(z)}{d z^{2}}\right) .
$$

Hence, the polynomials $B_{n}^{(r)}(z), n \geq 2$, satisfy the following second order differential equation

$$
2 z y^{\prime \prime}-(z-2 r+2 n-1) y^{\prime}+n y=0 .
$$

3.2 The zeros of the polynomials $B_{n}^{(n-s)}(z), s=1, \ldots, n-1$. Let $z_{n, 1}^{(n-s)}, z_{n, 2}^{(n-s)}, \cdots, z_{n, n}^{(n-s)}$ be the zeros of $B_{n}^{(n-s)}(z), n \geq 1, s=$ $1, \ldots, n-1$. We prove the following result.

Theorem 6. The polynomial $B_{n}^{(n-s)}(z), n \geq 1, s=1,2, \ldots, n-1$, has exactly $(n-s)$ positive and simple zeros.

Proof. By (34) the polynomial $B_{n}^{(n-s)}(z), n \geq 1$, can be written as a linear combination of the classical orthogonal polynomials $B_{n-s}^{(n-s)}(z), \ldots, B_{n-1}^{(n-1)}(z), B_{n}^{(n)}(z)$. So, it has at least $n-s$ positive and distinct zeros.

If we write $B_{n}^{(n-s)}(z)=\sum_{i=0}^{n} b_{n, i}^{(n-s)} z^{i}$, then from (33) we have

$$
b_{n, i}^{(n-s)}=(-1)^{n-i}\left(\begin{array}{c}
n \\
i
\end{array}\right) \frac{(2(n-s)-1) ! !}{(2(i-s)-1) ! !}, \quad 0 \leq i \leq n .
$$

If $i \leq s$, then

$$
(2(i-s)-1) ! !=(-[2(s-i)+1]) ! !=\frac{(-1)^{s-i}}{(2(s-i)-1) ! !} .
$$

Hence,

$$
b_{n, i}^{(n-s)}=(-1)^{n-s}\left(\begin{array}{c}
n \\
i
\end{array}\right)(2(n-s)-1) ! !(2(s-i)-1) ! !, \quad 0 \leq i \leq s .
$$


It follows that

$$
\operatorname{sgn}\left(b_{n, i}^{(n-s)}\right)=(-1)^{n-s}, \quad 0 \leq i \leq s .
$$

On the other hand, if $i>s$ we have $\operatorname{sgn}\left(b_{n, i}^{(n-s)}\right)=(-1)^{n-i}$.

By Descartes' rule of signs, $B_{n}^{(n-s)}(z), n \geq 1, s=1,2, \ldots, n-1$, has at most $n-s$ positive zeros. Hence, $B_{n}^{(n-s)}(z), n \geq 1, s=1,2, \ldots, n-1$, has exactly $n-s$ positive and simple zeros.

From the last theorem we can conclude that the remaining $s$ zeros of $B_{n}^{(n-s)}(z), s=1, \ldots, n-1$, are negative or they occur in complex conjugate pairs. Thus, if $s$ is odd, there is at least one negative zero of $B_{n}^{(n-s)}(z)$. So, the polynomial $B_{n}^{(n-1)}(z), n=1,2, \ldots$, has $n-1$ positive and simple zeros and one negative zero, $z_{n, 1}^{(n-1)}$ say.

From (35) we obtain the following identities

$$
n b_{n-1, i}^{(r)}=(i+1) b_{n, i+1}^{(r)}, \quad i=0,1, \ldots, n-1 .
$$

Hence, from (36) and (37) we have

$$
\operatorname{sgn}\left(B_{n}^{(n-2)}(0)\right)=\operatorname{sgn}\left(B_{n-1}^{(n-2)}(0)\right)=(-1)^{n} .
$$

Let $z_{n-1,1}^{(n-2)}$ be the negative zero of $B_{n-1}^{(n-2)}(z)$. From the recurrence relation (10) and also from (28) we obtain

$$
B_{n}^{(n-2)}\left(z_{n-1,1}^{(n-2)}\right)=-2(n-1) z_{n-1,1}^{(n-2)} B_{n-2}^{(n-2)}\left(z_{n-1,1}^{(n-2)}\right) .
$$

Then,

$$
\operatorname{sgn}\left(B_{n}^{(n-2)}\left(z_{n-1,1}^{(n-2)}\right)\right)=\operatorname{sgn}\left(B_{n-2}^{(n-2)}\left(z_{n-1,1}^{(n-2)}\right)\right) .
$$

Since all the zeros of $B_{n-2}^{(n-2)}(z)$ are positive, from (36) and (37) we have

$$
\operatorname{sgn}\left(B_{n-2}^{(n-2)}\left(z_{n-1,1}^{(n-2)}\right)\right)=\operatorname{sgn}\left(B_{n-2}^{(n-2)}(0)\right)=\operatorname{sgn}\left(B_{n-1}^{(n-2)}(0)\right) .
$$


From (38),

$$
\operatorname{sgn}\left(B_{n}^{(n-2)}\left(z_{n-1,1}^{(n-2))}\right)=\operatorname{sgn}\left(B_{n}^{(n-2)}(0)\right)=(-1)^{n} .\right.
$$

Moreover,

$$
\operatorname{sgn}\left(B_{n-1}^{(n-2)}(z)\right)=(-1)^{n-1} \quad \text { if } z \in\left(-\infty, z_{n-1,1}^{(n-2)}\right)
$$

and

$$
\operatorname{sgn}\left(B_{n-1}^{(n-2)}(z)\right)=(-1)^{n} \quad \text { if } z \in\left(z_{n-1,1}^{(n-2)}, 0\right) .
$$

Suppose $n$ odd. In this case, from (35) we can conclude that the polynomial $B_{n}^{(n-2)}(z)$ is increasing for $z \in\left(-\infty, z_{n-1,1}^{(n-2)}\right)$ and it is decreasing for $z \in\left(z_{n-1,1}^{(n-2)}, 0\right)$. Then, from $(39), B_{n}^{(n-2)}(z)<0$ for $z \leq 0$ and so $B_{n}^{(n-2)}(z)$ has $n-2$ simple and positive zeros and a pair of complex conjugate zeros.

Similarly, $B_{n-1}^{(n-3)}(z)>0$ if $z \leq 0$. Then, from $(35), B_{n}^{(n-3)}(z)$ is an increasing function if $z \leq 0$. However,

$$
\lim _{z \rightarrow-\infty} B_{n}^{(n-3)}(z)=-\infty
$$

and, from (36) and (37), $B_{n}^{(n-3)}(0)>0$. Hence, there is only one negative zero of $B_{n}^{(n-3)}(z)$.

By mathematical induction we conclude the following result.

Theorem 7. The polynomial $B_{n}^{(n-s)}(z), n \geq 1, s=1, \ldots, n-1$, satisfies:

(i) if $s$ is an odd integer, $B_{n}^{(n-s)}(z)$ has one negative zero and $s-1$ complex zeros;

(ii) if $s$ is an even integer, $B_{n}^{(n-s)}(z)$ has $s$ complex zeros.

The following theorem concerns the positive zeros of the polynomials $B_{n}^{(n-s)}(z), s=1, \ldots, n-1, n \geq 1$. 
Theorem 8. The positive zeros of the polynomials $B_{n}^{(n-s+1)}(z)$ and $B_{n}^{(n-s)}(z), n \geq 2, s=1, \ldots, n-1$, interlace.

Proof. Given that $\left\{B_{n}^{(n)}(z)\right\}_{n=0}^{\infty}$ is a sequence of orthogonal polynomials with respect to the distribution $d \psi(t)$, the zeros of $B_{n}^{(n)}(z)$ and $B_{n-1}^{(n-1)}(z), n=2,3, \ldots$, interlace. Then, for $i=1, \cdots, n-1$

$$
\operatorname{sgn}\left(B_{n-1}^{(n-1)}\left(z_{n, i}^{(n)}\right)\right)=-\operatorname{sgn}\left(B_{n-1}^{(n-1)}\left(z_{n, i+1}^{(n)}\right)\right) .
$$

Let $z_{n, i}^{(n-s)}, i=s+1, \ldots, n$, be the positive zeros of $B_{n}^{(n-s)}(z)$ and, if $s$ is odd, let $z_{n, s}^{(n-s)}$ be the negative zero.

From (12) and (28)

$$
B_{n}^{(n-1)}\left(z_{n, i}^{(n)}\right)=2 n B_{n-1}^{(n-1)}\left(z_{n, i}^{(n)}\right), \quad i=1, \ldots, n .
$$

Thus,

$$
\operatorname{sgn}\left(B_{n}^{(n-1)}\left(z_{n, i}^{(n)}\right)\right)=\operatorname{sgn}\left(B_{n-1}^{(n-1)}\left(z_{n, i}^{(n)}\right)\right), \quad i=1, \ldots, n .
$$

Since $B_{n}^{(n-1)}(z)$ has $(n-1)$ positive and simple zeros, from (40) and the last equation we obtain

$$
z_{n, i+1}^{(n-1)} \in\left(z_{n, i}^{(n)}, z_{n, i+1}^{(n)}\right), \quad i=1, \ldots, n-1 .
$$

Thus, Theorem 8 holds for $s=1$.

Suppose that the positive zeros of $B_{n}^{(n-s+1)}(z)$ and $B_{n}^{(n-s)}(z)$ interlace for all integer $s, 1 \leq s<n-1$.

Then, for $i=s+1, \cdots, n-1$

$$
\operatorname{sgn}\left(B_{n}^{(n-s+1)}\left(z_{n, i}^{(n-s)}\right)\right)=-\operatorname{sgn}\left(B_{n}^{(n-s+1)}\left(z_{n, i+1}^{(n-s)}\right)\right) .
$$

From equation (13) with $r=n-s$, we obtain

$$
B_{n}^{(n-s-1)}\left(z_{n, i}^{(n-s)}\right)=\frac{-z_{n, i}^{(n-s)}}{\alpha_{n+1}^{(n-s)}+\beta_{n+1}^{(n-s)}} B_{n}^{(n-s+1)}\left(z_{n, i}^{(n-s)}\right),
$$


for $i=1, \ldots, n$. Since $\alpha_{n+1}^{(n-s)}+\beta_{n+1}^{(n-s)}=2(n-s)-1>0$, from $(42)$ and (43) we have

$$
\operatorname{sgn}\left(B_{n}^{(n-s-1)}\left(z_{n, i}^{(n-s)}\right)\right)=-\operatorname{sgn}\left(B_{n}^{(n-s-1)}\left(z_{n, i+1}^{(n-s)}\right)\right)
$$

for $i=s+1, \ldots, n-1$. Hence,

$$
z_{n, i+1}^{(n-s-1)} \in\left(z_{n, i}^{(n-s)}, z_{n, i+1}^{(n-s)}\right), \quad i=s+1, \ldots, n-1 .
$$

$\square$

Similarly we can show that

$$
z_{n, i+1}^{(n-1)} \in\left(z_{n-1, i}^{(n-1)}, z_{n, i+1}^{(n)}\right), \quad i=1,2, \ldots, n-1 .
$$

Finally, we investigate the behavior of the single negative zero of the polynomials $B_{n}^{(n-s)}(z)$ when $s \leq n$ is an odd integer.

Consider then $s$ an odd integer between 3 and $n$. The polynomials $B_{n}^{(n-s+2)}(z)$ and $B_{n}^{(n-s)}(z)$ each have one negative zero, $z_{n, s-2}^{(n-s+2)}$ and $z_{n, s}^{(n-s)}$ respectively. The polynomial $B_{n}^{(n-s+1)}(z)$ has $n-s+1$ positive zeros and $s-1$ complex zeros. Hence, from (13), we obtain

$$
B_{n}^{(n-s)}\left(z_{n, s-2}^{(n-s+2)}\right)=\frac{\beta_{n+1}^{(n-s+1)}+z_{n, s-2}^{(n-s+2)}}{\alpha_{n+1}^{(n-s+1)}+\beta_{n+1}^{(n-s+1)}} B_{n}^{(n-s+1)}\left(z_{n, s-2}^{(n-s+2)}\right) .
$$

Suppose $n$ is odd. Then, $B_{n}^{(n-s+1)}(z) \rightarrow-\infty$ as $z \rightarrow-\infty$. Thus,

$$
B_{n}^{(n-s+1)}\left(z_{n, s-2}^{(n-s+2)}\right)<0 .
$$

Since $\alpha_{n+1}^{(n-s+1)}+\beta_{n+1}^{(n-s+1)}=2(n-s)+1>0$ and $\beta_{n+1}^{(n-s+1)}=$ $-2 s+1<0$, from equation (44) we obtain $B_{n}^{(n-s)}\left(z_{n, s-2}^{(n-s+2)}\right)>0$. However, $\lim _{z \rightarrow-\infty} B_{n}^{(n-s)}(z)=-\infty$.

Hence,

$$
z_{n, s}^{(n-s)}<z_{n, s-2}^{(n-s+2)},
$$


and we conclude that the sequence of negative zeros $\left\{z_{n, s}^{(n-s)}\right\}, s=$ $1,3, \ldots, n$, is decreasing.

If $n$ is even the conclusion is the same for $s=1,3, \ldots, n-1$.

\section{REFERENCES}

1. C.F. Bracciali and J.H. McCabe Some extensions of M-fractions related to strong Stieltjes distribution, in Rational approximation (A. Cuyt and B. Verdonk, eds.), Acta Appl. Math. 61 (2000), 65-80.

2. C.F. Bracciali, J.H. McCabe and A. Sri Ranga On a symmetry in strong distributions, J. Comput. Appl. Math. 105 (1999), 187-198.

3. T. Chihara, An introduction to orthogonal polynomials, in Mathematics and its applications, vol. 13, Gordon \& Breach, New York, 1978.

4. J.H. McCabe, A continued fraction expansion with a truncation error estimate, for Dawson's integral, Math. Comp. 28 (1974), 811-816.

5. — A formal extension of the Padé table to include two-point Padé quotients, J. Inst. Math. Appl. 15 (1975), 363-372.

6. - On the even extension of an $M$-fraction, in Padé approximation and its applications, Conf. Proceedings (Amsterdam, 1980) (M.G. de Bruin and H. van Rossum, eds.), vol. 888, Lecture Notes in Math., Springer-Verlag, Berlin/New York, 1981, pp. 290-299.

7. - The quotient-difference and the Padé table: An alternative form and a general continued fraction, Math. Comp. 41 (1983), 183-197.

8. A. Sri Ranga, Continued fractions which correspond to two series expansions, and the strong Hamburger moment problem, Ph.D. Thesis, University of St. Andrews, St. Andrews, Scotland, UK, 1993.

9. A. Sri Ranga, E.X.L. de Andrade and J.H. McCabe, Some consequences of a symmetry in strong distributions, J. Math. Anal. Appl. 193 (1995), 158-168.

10. G. Szegö, Orthogonal polynomials, Amer. Math. Soc. Colloq. Publ., vol. 23, 4th ed., AMS, Providence, RI, 1975.

Departamento de Ciências de ComputaÇão e Estatística, ibilce, Universidade Estadual Paulista, Rua Cristóvão Colombo, 2265, 15054-000 SÃo José do Rio Preto (SP), Brazil

E-mail address: eliana@dcce.ibilce.unesp.br

Mathematical Institute, University of St. Andrews, St. Andrews, Fife KY16 9SS, Scotland, UK

E-mail address: jhm@st-andrews.ac.uk 